\title{
Cryo Transmission Electron Microscopy of Three Research-Grade Liposomal Formulations of Doxorubicin
}

\author{
William Monroe ${ }^{1}$, Angel Paredes ${ }^{1}$, Patrick Sisco ${ }^{2}$, Kristen Ahlschwede ${ }^{1}$ and Sean Linder ${ }^{2}$ \\ 1. U.S. Food and Drug Administration, National Center for Toxicological Research, Jefferson, Arkansas, \\ USA \\ 2. U.S. Food and Drug Administration, Office of Regulatory Affairs, Jefferson, Arkansas, USA
}

Nanotechnology has sparked a rapidly growing interest in medicine with the potential to solve a number of issues associated with conventional therapeutics, including poor water solubility, lack of targeting capability, nonspecific distribution, systemic toxicity, and low therapeutic index. Over the past several decades, remarkable progress has been made in the development and application of engineered nanoparticles to treat diseases more effectively. One of these key nanomaterials is the liposome, which is currently being used to encapsulate various chemotherapeutic agents. This encapsulation is engineered to increase the efficacy of the chemotherapeutic agent by protecting it against degradation, prolonging circulation time, improving cellular uptake, and reducing side effects that limit dosage amounts. As the commercialization of liposomes has increased, regulatory-based methodologies for their characterization have not been well documented. As such, to ensure the safety and quality of commercialized liposomal products, the development of methodologies to characterize these materials is imperative [1].

In this work, we address this concern by investigating a method to characterize the particle size and size distribution associated with liposome based drugs. More specifically, this study uses three researchgrade liposomal formulations of doxorubicin with sizes of 100, 200, and $300 \mathrm{~nm}$. Particle size and size distribution were analyzed using electron cryomicroscopy (cryoEM) which is an established technique that preserves small biomolecular structures in their most native state. The $100 \mathrm{~nm}$ liposomes were confirmed to be monodisperse and close to their expected size.

The cryoEM image in Figure 1 shows that the $200 \mathrm{~nm}$ liposomal formulation is polydispersed and larger than anticipated. These results were similar to those obtained by dynamic light scattering (DLS). These methods and results will guide the scientists in manufacturing and regulatory agencies to verify the formulation characteristics and ensure batch to batch consistency. These three formulations will be used as test material for future pharmacokinetic studies [2].

The information in these materials is not a formal dissemination of information by the FDA and does not represent agency position or policy. 


\section{References:}

[1] Report by the Arkansas Regional Laboratory, U.S. Food and Drug Administration, July, 2014 [2] Gabizon, A., H. Shmeeda, and Y. Barenholz, Pharmacokinetics of pegylated liposomal Doxorubicin: review of animal and human studies. Clin Pharmacokinet, 2003. 42(5): p.419-36
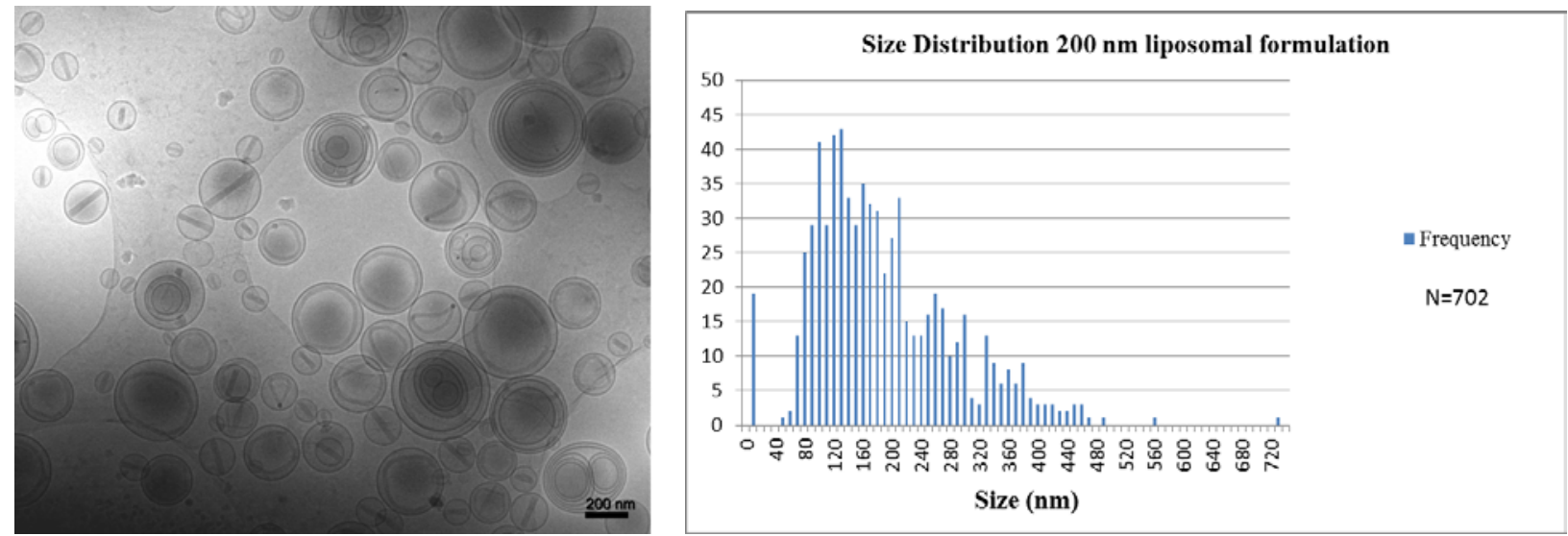

Figure 1. Left. Representative CryoEM image of $200 \mathrm{~nm}$ liposomal doxorubicin formulation. Right. Size distribution of the same sample. Scale bar $=200 \mathrm{~nm}$. 